\title{
Dhh-expressing Schwann cell precursors contribute to skin and cochlear melanocytes, but not to vestibular melanocytes
}

Running title: Melanocyte-forming potential of Dhh-expressing SCPs

Grégoire Bonnamour ${ }^{1,2}$, Rodolphe Soret ${ }^{1,2}$ and Nicolas Pilon ${ }^{1,2,3, *}$

${ }^{1}$ Molecular Genetics of Development Laboratory, Département des Sciences Biologiques, Université du Québec à Montréal (UQAM), Montréal H3C 3P8, Québec, Canada.

${ }^{2}$ Centre d'excellence en recherche sur les maladies orphelines - Fondation Courtois (CERMO-FC), Université du Québec à Montréal, Montréal H2X 3Y7, Québec, Canada.

${ }^{3}$ Département de pédiatrie, Université de Montréal, Montréal H3T 1C5, Québec, Canada.

"Corresponding Author: pilon.nicolas@uqam.ca

\begin{abstract}
For a long time, melanocytes were believed to be exclusively derived from neural crest cells migrating from the neural tube toward the developing skin. This notion was then challenged by studies suggesting that melanocytes could also be made from neural crest-derived Schwann cell precursors (SCPs) on peripheral nerves. A SCP origin was inferred from lineage tracing studies in mice using a Plp1 promotercontrolled Cre driver transgene (Plp1-CreERT2) and a fluorescent Rosa26 locus-controlled Cre reporter allele (Rosa26 FloxSTOP-YFP $)$. However, doubts were raised in part because another SCP-directed Cre driver controlled by the $D h h$ promoter ( $D h h$-Cre) was apparently unable to label melanocytes when used with a

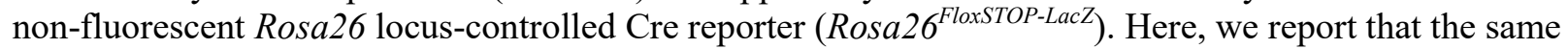
Dhh-Cre driver line can efficiently label melanocytes when used in a pure FVB/N background together with the fluorescent instead of the non-fluorescent Rosa26 locus-controlled Cre reporter. Our data further suggest that the vast majority of skin melanocytes are SCP-derived. Interestingly, we also discovered that SCPs contribute inner ear melanocytes in a region-specific manner, extensively contributing to the cochlea but not to the vestibule.
\end{abstract}

SIGNIFICANCE: This study validates the concept that an important subset of skin melanocytes are derived from neural crest cells through a Schwann cell precursor intermediate stage. Our data further allow to extend this concept for inner ear melanocytes located in the cochlea. These findings may thus be of clinical relevance for understanding the pathogenic mechanisms of both pigmentation and hearing disorders.

KEYWORDS: Schwann cell precursors; Dhh; Plp1; Cre/LoxP; Genetic cell lineage tracing; Skin melanocytes, Inner ear melanocytes; Cochlea; Vestibule; Mouse models.

ACKNOWLEDGEMENTS: The authors thank Denis Flipo (Cellular analyses and Imaging core, CERMOFC, UQAM) for assistance with confocal imaging. Funding of this work was provided via a grant from the Natural Science and Engineering Research Council of Canada (NSERC) to NP (\#RGPIN-2019-07076). GB was also supported by a studentship from the CERMO-FC, whereas NP is a FRQS senior research scholar and the recipient of the UQAM Research Chair on rare genetic diseases.

CONFLICT OF INTEREST: The authors declare no conflict of interest. 
The neural crest is a transient, vertebrate-specific cell population of the developing neural tube that generates a wide variety of specialized cell types such as craniofacial chondrocytes, peripheral neurons, peripheral nerve-associated Schwann cells, and melanocytes of the skin and inner ear (Dupin, Calloni, Coelho-Aguiar, \& Le Douarin, 2018). To generate these diverse cell types, the multipotent neural crest cells emigrate from the dorsal neural tube as a wave that begins in the cranial region of the embryo and that then spreads along the anterior-posterior axis (Szabo \& Mayor, 2018), in a coordinated manner with axial elongation and neural tube closure. The differentiation potential of neural crest cells is influenced by their axial origin, on the basis of which four main subpopulations of neural crest cells can be defined (from anterior to posterior): cranial, vagal, trunk and sacral. Although each subpopulation generates its own set of derivatives (Soldatov et al., 2019), all four subpopulations share the ability to form cells of the melanoblast/melanocyte lineage expressing specific markers such as MITF (Microphthalmia-associated transcription factor), KIT (Mast/stem cell growth factor receptor KIT) and DCT (Dopachrome tautomerase) (Mort, Jackson, \& Patton, 2015).

Analysis of the trunk subpopulation in mouse and chick embryos has revealed the existence of two migration pathways. One known as the "ventral pathway" passes through the somites to give rise to various neural derivatives (e.g., sensory neurons in dorsal root ganglia and Schwann cells along peripheral nerve tracts), while the other one known as the "dorsolateral pathway" runs under the surface ectoderm to specifically give rise to skin melanocytes (Mort et al., 2015; Szabo \& Mayor, 2018). This dorsolateral route has long been considered the unique provider of melanocytes, a concept that was challenged over the past decade by experimental evidences in chick and mouse suggesting another neural crest contribution by ventrally migrating Schwann cell precursors (SCPs) (Adameyko et al., 2009; Adameyko et al., 2012; Nitzan, Pfaltzgraff, Labosky, \& Kalcheim, 2013).

An SCP origin of melanocytes was deduced from cell lineage tracing studies in mice (Adameyko et al., 2009; Adameyko et al., 2012), which were based on a single genetic system involving the tamoxifen inducible Cre driver Plp1-CreERT2 (Leone et al., 2003) and the fluorescent Cre reporter Rosa26 $6^{\text {FloxSTOP-YFP (Srinivas et al., }}$ 2001). These studies showed that a single tamoxifen dose at embryonic day (e)11.0 was sufficient to yield YFP-positive (i.e., derived from Plp1-expressing cells) melanoblasts/melanocytes in the trunk (Adameyko et al., 2009), whereas an earlier pulse at e9.5 yielded YFP-positive melanoblasts/melanocytes in the head (Adameyko et al., 2012). Yet, some doubts about the specificity of the Plp1-CreERT2 tool for the SCP lineage were then raised following studies that reported wider Cre protein expression in non-SCP-fated neural crest cells at e9.5 (Hari et al., 2012), and specific Plp1 gene expression in e15.5 melanoblasts recovered by FACS (fluorescence-activated cell sorting) from the skin of Tyr-Cre;Z/EG double transgenic embryos (Colombo, Champeval, Rambow, \& Larue, 2012).

The above controversy was also fueled by the apparent inefficiency of the alternative Cre driver Dhh-Cre to label SCP-derived melanoblasts/melanocytes (Hari et al., 2012; Radomska et al., 2019; Wong et al., 2006), although no study unambiguously showed that Dhh-expressing SCPs are not capable of generating melanocytes. The Dhh-Cre driver is believed to faithfully replicate endogenous Dhh expression in developing 
Schwann, Sertoli and endothelial cell lineages (Bitgood \& McMahon, 1995; Jaegle et al., 2003; Parmantier et al., 1999). In developing Schwann cells, the Dhh-Cre transgene is supposedly active from e11.5 to postnatal day (P) 10 (Bitgood \& McMahon, 1995; Jaegle et al., 2003; Parmantier et al., 1999; Sharghi-Namini et al., 2006), and this Cre driver has notably proved useful for demonstrating the SCP origin of a subset of enteric neurons (Soret et al., 2020; Uesaka, Nagashimada, \& Enomoto, 2015). The apparent discrepancy in melanocyte-labeling capacity between Plp1-CreERT2 and Dhh-Cre should thus be interpreted with caution. Although such difference might potentially be explained by the existence of different subpopulations of SCPs, as suggested from studies about the SCP contribution to skeleton formation (Xie et al., 2019), it might also simply be due to experimental differences. Notably, previous studies that directly verified the existence of SCP-derived melanoblasts/melanocytes with Dhh-Cre at prenatal (Hari et al., 2012) and postnatal (Wong et al., 2006) stages were both performed using the Cre reporter Rosa $26^{\text {FloxSTOP-LacZ }}$ and enzymatic detection of $\beta$ Galactosidase activity (Soriano, 1999) instead of the Cre reporter Rosa26 FloxSTOP-YFP and presumably more sensitive detection of YFP fluorescence (Srinivas et al., 2001). In postnatal Dhh-Cre; Rosa $26^{\text {FloxSTOP-LacZ }}$ mice, a weak staining reaction would be a plausible explanation for the apparent contradictory findings that $\beta$ Galactosidase activity was detected in unpigmented SOX10/p75-positive cells from the bulge region of hair follicles (consistent with a melanocyte stem cell identity), but not in darkly pigmented mature melanocytes from the bulb region (Wong et al., 2006). Moreover, in both of these previous studies (from the same group) the Dhh-Cre driver was apparently used in a mixed genetic background of undefined nature (as deduced from the presence of pigmented melanocytes in Wong et al., 2006) instead of the albino FVB/N genetic background of origin for this line (Jaegle et al., 2003). Switching FVB/N to a mixed genetic background might have caused inaccurate expression of the Dhh-Cre driver and thereby erroneous cell lineage tracing, as previously reported for a $C d x 2$ enhancer-driven Cre transgene (Coutaud \& Pilon, 2013). The FVB/N genetic background also offers the advantage of facilitating marker analyses in mature melanocytes by preventing interference from dark melamine pigments, which are absent in this albino background.

In the course of studies aimed at determining the origin of inner ear melanocytes, we decided to re-evaluate the potential of $D h$-expressing SCPs to generate melanocytes, but now using the same fluorescent Cre reporter (Rosa26 $6^{\text {FloxSTOP-YFP }}$ ) previously used to analyze the contribution of Plpl-expressing SCPs. This interest for inner ear melanocytes was motivated by our prior work with the Spot mouse model of Waardenburg syndrome type 4, which suggested that melanocytes from the cochlear and vestibular compartments of the inner ear might have distinct origins (Bergeron et al., 2016).

All mice used in the current study were maintained in the albino FVB/N genetic background (after backcrossing for at least 5 generations when mice had a different background of origin). The Dhh-Cre [Jax stock \#012929; FVB(Cg)-Tg(Dhh-cre)1Mejr/J] (Jaegle et al., 2003) and Plp1-CreERT [Jax stock \#005975; B6.Cg-Tg(Plp1-cre/ERT)3Pop/J (Doerflinger, Macklin, \& Popko, 2003) lines were purchased from The Jackson Laboratory, whereas the Rosa26 $6^{\text {FloxSTOP-YFP }}$ line [Gt(ROSA)26Sor ${ }^{\text {tml(EYFP)Cos}}$ (Srinivas et al., 2001) was directly provided by Dr. Frank Costantini (Columbia University). All manipulations were performed in 
accordance with the guidelines of the Canadian Council of Animal Care as approved by the Comité institutionnel de protection des animaux (CIPA reference \#899) of Université du Québec à Montréal.

Dhh-Cre;Rosa2 $6^{\text {FloxSTOP-YFP }}$ double transgenic mice were first evaluated for the presence of YFP-positive and Cre-negative melanocytes in $1 \mathrm{~cm}^{2}$ pieces of skin from the back and belly at postnatal day (P)12 (i.e., during anagen phase of the hair cycle). Sciatic nerves were also used as positive control for Cre expression. Some of the freshly-dissected pieces of skin were immediately used to directly analyze emission of YFP fluorescence (after further manual trimming to allow transversal imaging), whereas sciatic nerves and all other pieces of skin were processed for immunofluorescence as previously described (Bergeron et al., 2015). To this end, skin and sciatic nerve samples were fixed in $4 \%$ paraformaldehyde for $4 \mathrm{~h}$ on ice and washed $3 \times 5 \mathrm{~min}$ in PBS. For sectioning, skin samples were further incubated overnight in 30\% sucrose, embedded in OCT and cut at $20 \mu \mathrm{m}$ with a Leica CM1950 cryostat. Skin sections and whole sciatic nerves were then immunolabeled using standard blocking/washing/diluting solution (10\% fetal bovine serum and $0.1 \%$ Triton X-100 in PBS). Primary antibodies used were rabbit polyclonal anti-DCT (Abcam \# AB74073; diluted 1:200), goat polyclonal antiGFP/YFP (Abcam \#AB6673; diluted 1:200), and mouse monoclonal anti-Cre (Sigma \#Mab3120; diluted 1:500). Corresponding secondary antibodies were Alexa Fluor ${ }^{\circledR} 647$ AffiniPure donkey anti-rabbit IgG (Jackson Immunoresearch \# 711-605-152; diluted 1:500), Alexa Fluor ${ }^{\circledR} 488$ AffiniPure bovine anti-goat IgG (Jackson Immunoresearch \# 805-545-180; diluted 1:500), and Alexa Fluor ${ }^{\circledR} 594$ AffiniPure donkey antimouse IgG (Jackson Immunoresearch \# 715-585-151; diluted 1:500). All images were acquired with either a 20X (Plan Fluor 20x/0.75 Mimm) or a 60X (Plan Apo VC 60x1.40 oil) objective on a Nikon A1R confocal microscope.

Remarkably, analysis of immunolabeled pieces of back and belly skin at P12 (Fig.1A) revealed the presence of multiple YFP-DCT double-positive melanocytes (i.e., SCP-derived) in both hair follicles (Fig.1B-D) and dermis (Fig.1E). In hair follicles, YFP-DCT double-positive melanocytes were observed in both bulb and bulge compartments (Fig.1C-D), where mature melanocytes and melanocyte stem cells reside, respectively. None of these YFP-DCT double-positive melanocytes stained positive for Cre (Fig.1F-G), which was instead detected in YFP-positive but DCT-negative cells such as Schwann cells associated with cutaneous (Fig.1F arrow and inset) or sciatic (Fig. $1 \mathrm{H})$ nerves. About $85 \%$ of hair follicles were found to contain YFP-positive melanocytes, a proportion that remained virtually identical in both back and belly regions (Fig.1I). The average proportion of YFP-positive melanocytes per follicle was similar to the proportion of YFP-positive melanocytes in the dermis (Fig.1J), which were in both cases similar to the proportion of hair follicles containing YFP-positive melanocytes (Fig. 1I). These proportions of $85-90 \%$ are higher than the proportions of about $60-65 \%$ previously reported with the Plp1-CreERT2 driver upon exposure to a single pulse of tamoxifen (Adameyko et al., 2009). This difference is most likely due to the constitutively active nature of the Cre recombinase in the Dhh-Cre model, thereby suggesting that results with this Cre driver are probably more representative of the true extent of SCP contribution. Importantly, these results are not due to unspecific staining by the GFP/YFP antibody as we observed the same expression pattern when emission of YFP fluorescence was directly visualized in samples of unfixed skin (Fig.1K). 
To confirm the embryonic origin of skin melanocytes derived from $D h h$-expressing SCPs, we then analyzed their distribution in the trunk at e13.5. Dhh-Cre;Rosa26 $6^{\text {FloxSTOP-YFP }}$ double transgenic embryos were generated by natural mating, with noon of the day of vaginal plug detection being designated as e 0.5 . Cryosectionning, immunolabeling and imaging were performed as described above for postnatal skin, now focusing on transverse sections at forelimb level and also including a marker for peripheral nerves (mouse monoclonal anti-ßIII-Tubulin; Abcam \# AB78078; diluted 1:500).

In total agreement with our findings in the postnatal skin, we detected several YFP-DCT double-positive melanoblasts around the developing ventral ramus of $\beta$ III-Tubulin-positive peripheral nerves (Fig.2A). Once again, none of these SCP-derived melanoblasts was found to express Cre (Fig.2B). As expected, we also did not detect YFP expression in melanoblasts under the dorsolateral surface ectoderm that are presumably derived from the neural crest without passing through a SCP state (Fig.2C). In the wider dorsal region, YFP expression was instead exclusively confined to DCT-negative cells, which may include SCPs (as those associated with the boundary caps of both dorsal and ventral roots of spinal nerves) and endothelial cells that are both known to express Dhh at this developmental stage (Bitgood \& McMahon, 1995; Jaegle et al., 2003). Together with our observations in P12 skin, these results in e13.5 embryos are thus consistent with the notion that a majority of postnatal melanocytes is derived from the neural crest through an intermediary SCP state.

Having validated that SCP-derived melanocytes could be permanently labeled with YFP fluorescence in Dhh-Cre;Rosa $26^{\text {FloxSTOP-YFP }}$ mice, we finally used this model to analyze the potentially distinct origin of cochlear and vestibular melanocytes (Bergeron et al., 2016). For further validation, we also used Plp1CreERT;Rosa2 $6^{\text {FloxSTOP-YFP }}$ mice that were exposed to a single pulse of tamoxifen at e9.5 (via oral gavage of pregnant dams with $50 \mathrm{mg} / \mathrm{kg}$ tamoxifen and $2.5 \mathrm{mg}$ progesterone diluted in corn oil), a time point that was previously reported to allow tracing of melanoblasts derived from Plp1-expressing SCPs in the vicinity of the developing ear (Adameyko et al., 2012). In both cases, inner ears were microdissected from P12 double transgenic mice, fixed in $4 \% \mathrm{PFA}$ at $4^{\circ} \mathrm{C}$ overnight and demineralized in $0.5 \mathrm{M}$ EDTA (in PBS) during 5 days before being sectioned at $200 \mu \mathrm{m}$ with a vibrating blade microtome (Microm HM 650V, ThermoFisher). These sections were immunolabeled as described above for skin and embryo cross-sections, with the difference that melanocytes and YFP were now labeled using goat polyclonal anti-KIT (R\&D Systems \# AF1356; diluted 1:50) and rabbit polyclonal anti-GFP/YFP (Abcam \#AB290; diluted 1:100), respectively. Corresponding secondary antibodies were Alexa Fluor ${ }^{\circledR} 647$ AffiniPure bovine anti-goat IgG (Jackson Immunoresearch \# 805-605-18-0; diluted 1:500) and Alexa Fluor ${ }^{\circledR} 488$ donkey anti-rabbit IgG (Abcam \# AB150073; diluted $1: 500)$.

In line with our prior observations made with Spot mice (which are specifically lacking vestibular but not cochlear melanocytes (Bergeron et al., 2016)), both Dhh-Cre and Plp1-CreERT drivers were found to label melanocytes from P12 inner ears in a region-specific manner (Fig.3). In contrast to the crus commune (vestibule), where none of KIT-positive melanocytes were found to be YFP-positive (Fig.3A), the large majority of KIT-positive melanocytes from the stria vascularis (cochlea) were found to also express YFP

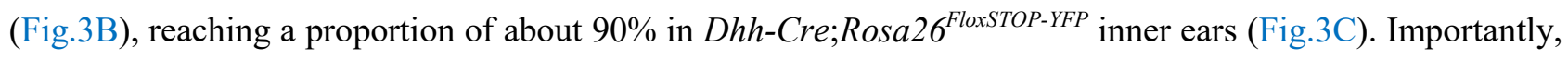


Cre expression from the Dhh-Cre transgene was only detected in nearby KIT-negative endothelial cells that make up small vessels and not in KIT-positive melanocytes, again validating the specificity of the system. These observations are thus in total agreement with the notion that cochlear and vestibular melanocytes come from two different pathways, with cochlear melanocytes mainly derived from SCPs and vestibular melanocytes derived from non-SCP-fated neural crest cells. This finding helps to explain the vestibule-restricted lack of melanocytes and the accompanying endolymphatic collapse that specifically leads to balance problems (without hearing loss) in the Spot mouse model of Waardenburg syndrome type 4 (Bergeron et al., 2016). SCPderived cochlear melanocytes are most likely coming from the cochleovestibular nerve, for which both the neuronal and glial compartments have been reported to invade the developing inner ear as early as at the e10.5 stage (Sandell, Butler Tjaden, Barlow, \& Trainor, 2014).

Overall, this study should help to resolve a long-standing controversy and instill confidence about the SCP origin of a majority of melanocytes, in both the skin and cochlea. Such knowledge should thus seriously be taken into consideration when analyzing the pathogenic mechanisms of pigmentation and inner ear anomalies. One especially interesting open question is whether the dorsolateral and ventral pathways are mutually influencing each other, in both health and disease states. Studies for addressing this clinically-relevant question will clearly be facilitated by current availability of a wide collection of spontaneous and genetically-engineered mouse models of pigmentation and/or inner ear disorders (Nakamura, Tobin, Richards-Smith, Sundberg, \& Paus, 2002; Pilon, 2016; Tachibana, Kobayashi, \& Matsushima, 2003). 


\section{REFERENCES}

Adameyko, I., Lallemend, F., Aquino, J. B., Pereira, J. A., Topilko, P., Muller, T., . . Ernfors, P. (2009). Schwann cell precursors from nerve innervation are a cellular origin of melanocytes in skin. Cell, 139(2), 366-379. doi:10.1016/j.cell.2009.07.049

Adameyko, I., Lallemend, F., Furlan, A., Zinin, N., Aranda, S., Kitambi, S. S., . . Ernfors, P. (2012). Sox2 and Mitf cross-regulatory interactions consolidate progenitor and melanocyte lineages in the cranial neural crest. Development, 139(2), 397-410. doi:10.1242/dev.065581

Bergeron, K. F., Cardinal, T., Toure, A. M., Beland, M., Raiwet, D. L., Silversides, D. W., \& Pilon, N. (2015). Male-Biased Aganglionic Megacolon in the TashT Mouse Line Due to Perturbation of Silencer Elements in a Large Gene Desert of Chromosome 10. PLoS Genet, 11(3), e1005093. doi:10.1371/journal.pgen.1005093

Bergeron, K. F., Nguyen, C. M., Cardinal, T., Charrier, B., Silversides, D. W., \& Pilon, N. (2016). Upregulation of the $\mathrm{Nr} 2 \mathrm{f1}-\mathrm{A} 830082 \mathrm{~K} 12 \mathrm{Rik}$ gene pair in murine neural crest cells results in a complex phenotype reminiscent of waardenburg syndrome type 4. Dis Model Mech, 9(11), 1283-1293. doi:10.1242/dmm.026773

Bitgood, M. J., \& McMahon, A. P. (1995). Hedgehog and Bmp genes are coexpressed at many diverse sites of cell-cell interaction in the mouse embryo. Dev Biol, 172(1), 126-138. doi:10.1006/dbio.1995.0010

Colombo, S., Champeval, D., Rambow, F., \& Larue, L. (2012). Transcriptomic analysis of mouse embryonic skin cells reveals previously unreported genes expressed in melanoblasts. J Invest Dermatol, 132(1), 170178. doi:10.1038/jid.2011.252

Coutaud, B., \& Pilon, N. (2013). Characterization of a novel transgenic mouse line expressing Cre recombinase under the control of the Cdx2 neural specific enhancer. Genesis, 51(11), 777-784. doi:10.1002/dvg.22421

Doerflinger, N. H., Macklin, W. B., \& Popko, B. (2003). Inducible site-specific recombination in myelinating cells. Genesis, 35(1), 63-72. doi:10.1002/gene.10154

Dupin, E., Calloni, G. W., Coelho-Aguiar, J. M., \& Le Douarin, N. M. (2018). The issue of the multipotency of the neural crest cells. Dev Biol, 444 Suppl 1, S47-S59. doi:10.1016/j.ydbio.2018.03.024

Hari, L., Miescher, I., Shakhova, O., Suter, U., Chin, L., Taketo, M., . . Sommer, L. (2012). Temporal control of neural crest lineage generation by Wnt/beta-catenin signaling. Development, 139(12), 21072117. doi:10.1242/dev.073064

Jaegle, M., Ghazvini, M., Mandemakers, W., Piirsoo, M., Driegen, S., Levavasseur, F., . . Meijer, D. (2003). The POU proteins Brn-2 and Oct-6 share important functions in Schwann cell development. Genes Dev, 17(11), 1380-1391. doi:10.1101/gad.258203

Leone, D. P., Genoud, S., Atanasoski, S., Grausenburger, R., Berger, P., Metzger, D., . . Suter, U. (2003). Tamoxifen-inducible glia-specific Cre mice for somatic mutagenesis in oligodendrocytes and Schwann cells. Mol Cell Neurosci, 22(4), 430-440. Retrieved from https://www.ncbi.nlm.nih.gov/pubmed/12727441

Mort, R. L., Jackson, I. J., \& Patton, E. E. (2015). The melanocyte lineage in development and disease. Development, 142(4), 620-632. doi:10.1242/dev.106567

Nakamura, M., Tobin, D. J., Richards-Smith, B., Sundberg, J. P., \& Paus, R. (2002). Mutant laboratory mice with abnormalities in pigmentation: annotated tables. J Dermatol Sci, 28(1), 1-33. doi:10.1016/s09231811(01)00158-x

Nitzan, E., Pfaltzgraff, E. R., Labosky, P. A., \& Kalcheim, C. (2013). Neural crest and Schwann cell progenitor-derived melanocytes are two spatially segregated populations similarly regulated by Foxd3. Proc Natl Acad Sci U S A, 110(31), 12709-12714. doi:10.1073/pnas.1306287110

Parmantier, E., Lynn, B., Lawson, D., Turmaine, M., Namini, S. S., Chakrabarti, L., . . Mirsky, R. (1999). Schwann cell-derived Desert hedgehog controls the development of peripheral nerve sheaths. Neuron, 23(4), 713-724. doi:10.1016/s0896-6273(01)80030-1

Pilon, N. (2016). Pigmentation-based insertional mutagenesis is a simple and potent screening approach for identifying neurocristopathy-associated genes in mice. Rare Diseases, 4(1), e1156287.

Radomska, K. J., Coulpier, F., Gresset, A., Schmitt, A., Debbiche, A., Lemoine, S., . . Topilko, P. (2019). Cellular Origin, Tumor Progression, and Pathogenic Mechanisms of Cutaneous Neurofibromas Revealed by Mice with Nf1 Knockout in Boundary Cap Cells. Cancer Discov, 9(1), 130-147. doi:10.1158/21598290.CD-18-0156 
Sandell, L. L., Butler Tjaden, N. E., Barlow, A. J., \& Trainor, P. A. (2014). Cochleovestibular nerve development is integrated with migratory neural crest cells. Dev Biol, 385(2), 200-210. doi:10.1016/j.ydbio.2013.11.009

Sharghi-Namini, S., Turmaine, M., Meier, C., Sahni, V., Umehara, F., Jessen, K. R., \& Mirsky, R. (2006). The structural and functional integrity of peripheral nerves depends on the glial-derived signal desert hedgehog. J Neurosci, 26(23), 6364-6376. doi:10.1523/JNEUROSCI.0157-06.2006

Soldatov, R., Kaucka, M., Kastriti, M. E., Petersen, J., Chontorotzea, T., Englmaier, L., . . Adameyko, I. (2019). Spatiotemporal structure of cell fate decisions in murine neural crest. Science, 364(6444). doi:10.1126/science.aas9536

Soret, R., Schneider, S., Bernas, G., Christophers, B., Souchkova, O., Charrier, B., . . Pilon, N. (2020). Glial Cell Derived Neurotrophic Factor Induces Enteric Neurogenesis and Improves Colon Structure and Function in Mouse Models of Hirschsprung Disease. Gastroenterology. doi:10.1053/j.gastro.2020.07.018

Soriano, P. (1999). Generalized lacZ expression with the ROSA26 Cre reporter strain. Nat Genet, 21(1), 7071. Retrieved from http://www.ncbi.nlm.nih.gov/entrez/query.fcgi?cmd=Retrieve\&db=PubMed\&dopt=Citation\&list_uids=9 916792

Srinivas, S., Watanabe, T., Lin, C. S., William, C. M., Tanabe, Y., Jessell, T. M., \& Costantini, F. (2001). Cre reporter strains produced by targeted insertion of EYFP and ECFP into the ROSA26 locus. BMC Dev Biol, 1, 4. Retrieved from http://www.ncbi.nlm.nih.gov/entrez/query.fcgi? $\mathrm{cmd}=$ Retrieve $\& \mathrm{db}=$ PubMed\&dopt=Citation\&list_uids=1 1299042

Szabo, A., \& Mayor, R. (2018). Mechanisms of Neural Crest Migration. Annu Rev Genet, 52, 43-63. doi:10.1146/annurev-genet-120417-031559

Tachibana, M., Kobayashi, Y., \& Matsushima, Y. (2003). Mouse models for four types of Waardenburg syndrome. Pigment Cell Res, 16(5), 448-454. Retrieved from http://www.ncbi.nlm.nih.gov/pubmed/12950719

Uesaka, T., Nagashimada, M., \& Enomoto, H. (2015). Neuronal Differentiation in Schwann Cell Lineage Underlies Postnatal Neurogenesis in the Enteric Nervous System. J Neurosci, 35(27), 9879-9888. doi:10.1523/JNEUROSCI.1239-15.2015

Wong, C. E., Paratore, C., Dours-Zimmermann, M. T., Rochat, A., Pietri, T., Suter, U., . . S Sommer, L. (2006). Neural crest-derived cells with stem cell features can be traced back to multiple lineages in the adult skin. $J$ Cell Biol, 175(6), 1005-1015. doi:10.1083/jcb.200606062

Xie, M., Kamenev, D., Kaucka, M., Kastriti, M. E., Zhou, B., Artemov, A. V., . . Chagin, A. S. (2019). Schwann cell precursors contribute to skeletal formation during embryonic development in mice and zebrafish. Proc Natl Acad Sci U S A. doi:10.1073/pnas.1900038116 


\section{FIGURE LEGENDS}

\section{Figure 1. Postnatal distribution of melanocytes derived from Dhh-expressing SCPs in the skin.}

(A) Pictures of P12 Dhh-Cre;Rosa26 $6^{\text {FloxSTOP-YFP }}$ double transgenic mice showing where pieces of back and belly skin were dissected. (B-H) Representative confocal microscopy images of $20 \mu \mathrm{m}$ transverse skin sections (B-G) or whole sciatic nerve $(\mathrm{H})$ immunolabeled with antibodies against DCT, YFP and/or Cre, and counterstained with DAPI (blue). DCT-YFP double-positive melanocytes are detected in both the bulb (filled arrowhead in B; zoom-in view in C) and bulge (empty arrowhead in B; zoom-in view in D) compartments of hair follicles, as well as in the dermis (arrowheads in the zoom-in view in E) where they are found scattered between cell-free (DAPI-negative) hair shafts. The arrow in the zoom-in view in E points to a DCT-positive but YFP-negative melanocyte, while the dotted line outlines the epidermis-dermis border and the asterisk indicates non-specific staining of external tissue border. DCT-YFP double-positive melanocytes do not express Cre, which can nonetheless be detected together with YFP in cutaneous (arrow in F; zoom-in view in inset) and sciatic $(\mathrm{H})$ nerve-associated Schwann cells. (I) Proportion of follicles containing YFP-positive melanocytes in back $(88.2 \% \pm 1.8 \% ; \mathrm{n}=388$ follicles from 5 animals $)$ and belly $(83.4 \% \pm 2.4 \% ; \mathrm{n}=180$ follicles from 5 animals) skin. (J) Proportion of YFP-positive melanocytes in dermis $(85.3 \% \pm 3.0 \% ; n=176$ DCT-positive dermal cells from 5 animals $)$, and both bulb $(89.8 \% \pm 1.1 \% ; \mathrm{n}=48$ bulbs from 5 animals $)$ and bulge $(87.8 \% \pm 4.5 \% ; n=15$ bulges from 5 animals) compartments of positive hair follicles. (K) Representative confocal microscopy image of direct YFP emission in a piece of unfixed dorsal skin along the transversal plane. Scale bar, $50 \mu \mathrm{m}$ (B, E right, H, K), $20 \mu \mathrm{m}(\mathrm{C}, \mathrm{D}), 100 \mu \mathrm{m}$ (E left, F), and $10 \mu \mathrm{m}(\mathrm{G})$.

\section{Figure 2. Prenatal distribution of melanoblasts derived from $D h h$-expressing SCPs at e13.5.}

(A-D) Representative confocal microscopy images of $20 \mu \mathrm{m}$ transverse sections (A-C) or whole embryos (D), taken from regions indicated in the schematic representation at the top ( $\mathrm{n} \geq 5$ for each region; NT, neural tube; DRG, dorsal root ganglion; VR, ventral ramus). Tissues were immunolabeled with antibodies against DCT, YFP and either $\beta$ III-Tubulin or Cre, and counterstained with DAPI (blue). DCT-YFP double-positive melanoblasts (arrowheads) are detected in the vicinity of $\beta$ III-Tubulin-positive ventral ramus (A, B; zoom-in views in insets) and in the developing skin (D). DCT-YFP double-positive melanoblasts do not express Cre, which can nonetheless be detected in ventral ramus-associated YFP-positive SCPs (B; arrows in left inset). Dorsolaterally located DCT-positive melanoblasts do not express YFP (C; zoom-in view in inset). Solid outlines delineate dorsal ramus (A, B) or neural tube, dorsal/ventral boundary caps, dorsal root ganglion and surface ectoderm (C). Scale bar, $100 \mu \mathrm{m}(\mathrm{A}, \mathrm{B}, \mathrm{D})$ or $50 \mu \mathrm{m}(\mathrm{C})$.

Figure 3. Postnatal distribution of melanocytes derived from Dhh-and Plp1-expressing SCPs in the inner ear. (A-B) Representative confocal microscopy images of $200 \mu \mathrm{m}$ sections of P12 inner ears, taken from regions indicated in the schematic representation on the left $(n=6$ for each region; SCC, semi-circular canal; 
CC, crus commune; RM, Reissner's membrane; SV, stria vascularis). Tissues were immunolabeled with antibodies against KIT, YFP and/or Cre, and counterstained with DAPI (blue). In both Dhh-Cre;Rosa26 FloxSTOP-

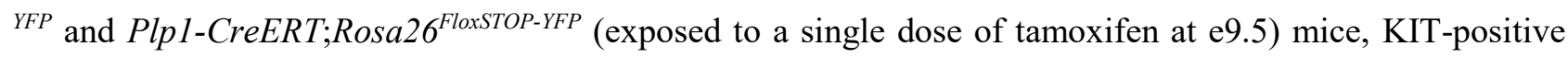
melanocytes express YFP in the stria vascularis (filled arrowheads in B) but not in the crus commune (empty arrowheads in A). KIT-YFP double-positive melanocytes from Dhh-Cre;Rosa26 $6^{\text {FloxSTOP-YFP }}$ mice do not express Cre, which can nonetheless be detected in endothelial cells (arrows in B). (C) Proportion of YFPpositive melanocytes in the stria vascularis of Dhh-Cre;Rosa $26^{\text {FloxSTOP-YFP }}$ mice $(88.8 \% \pm 4.0 \% ; \mathrm{n}=265 \mathrm{KIT}$ positive cells from 6 animals). Solid outlines in B delineate Reissner's membrane and associated cavities. Scale bar, $100 \mu \mathrm{m}$. 
Figure 1
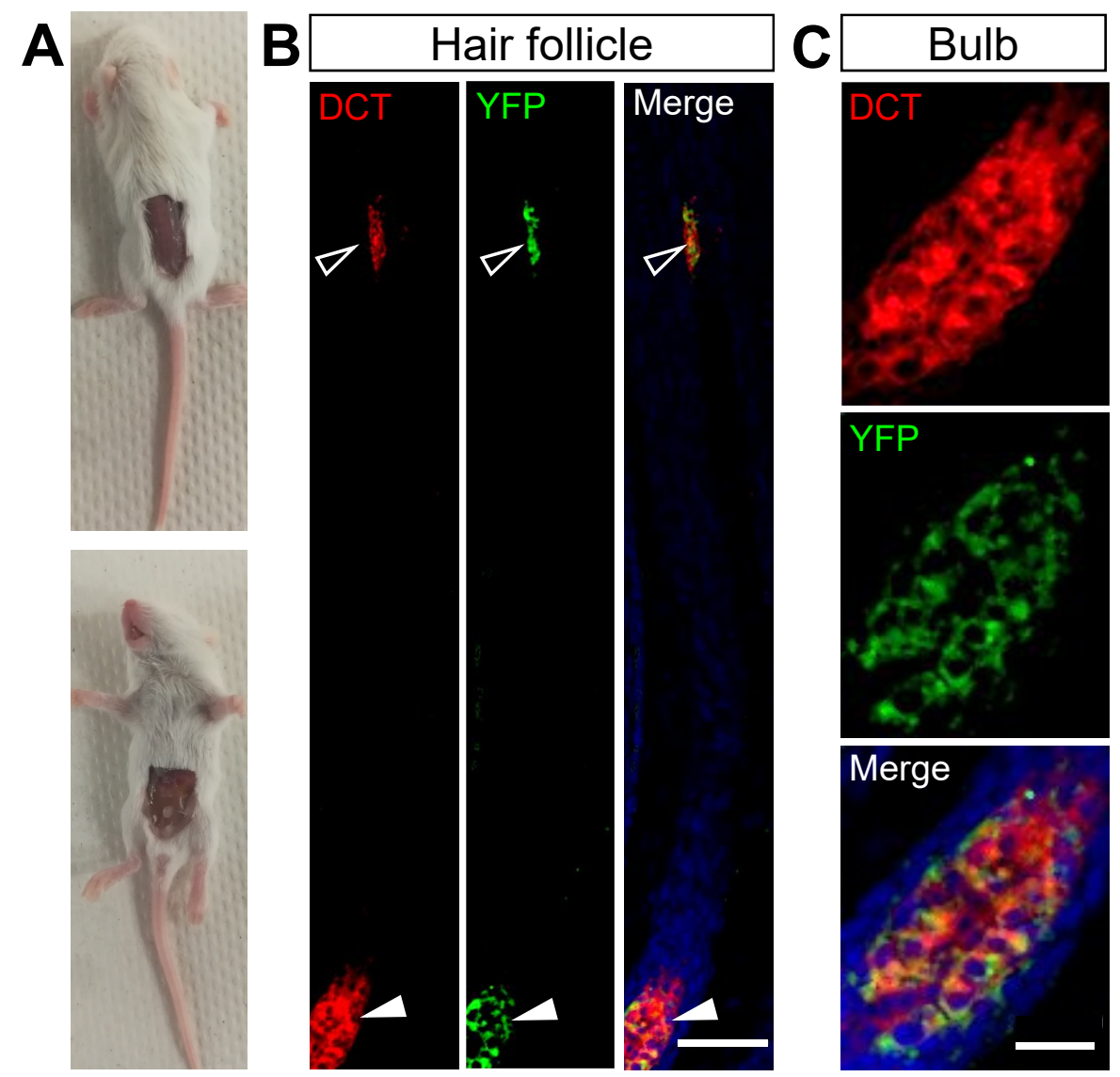

D Bulge
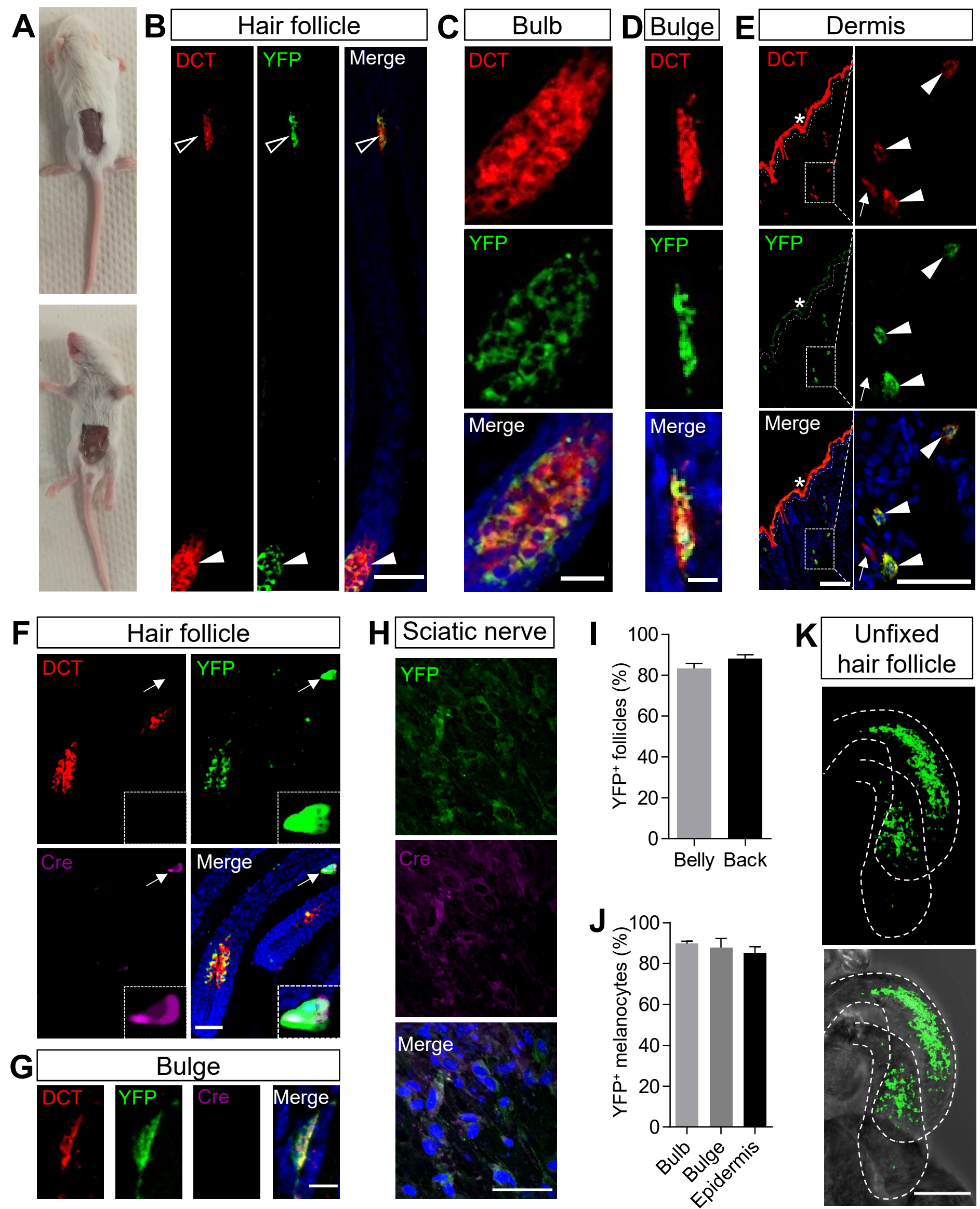
Figure 2

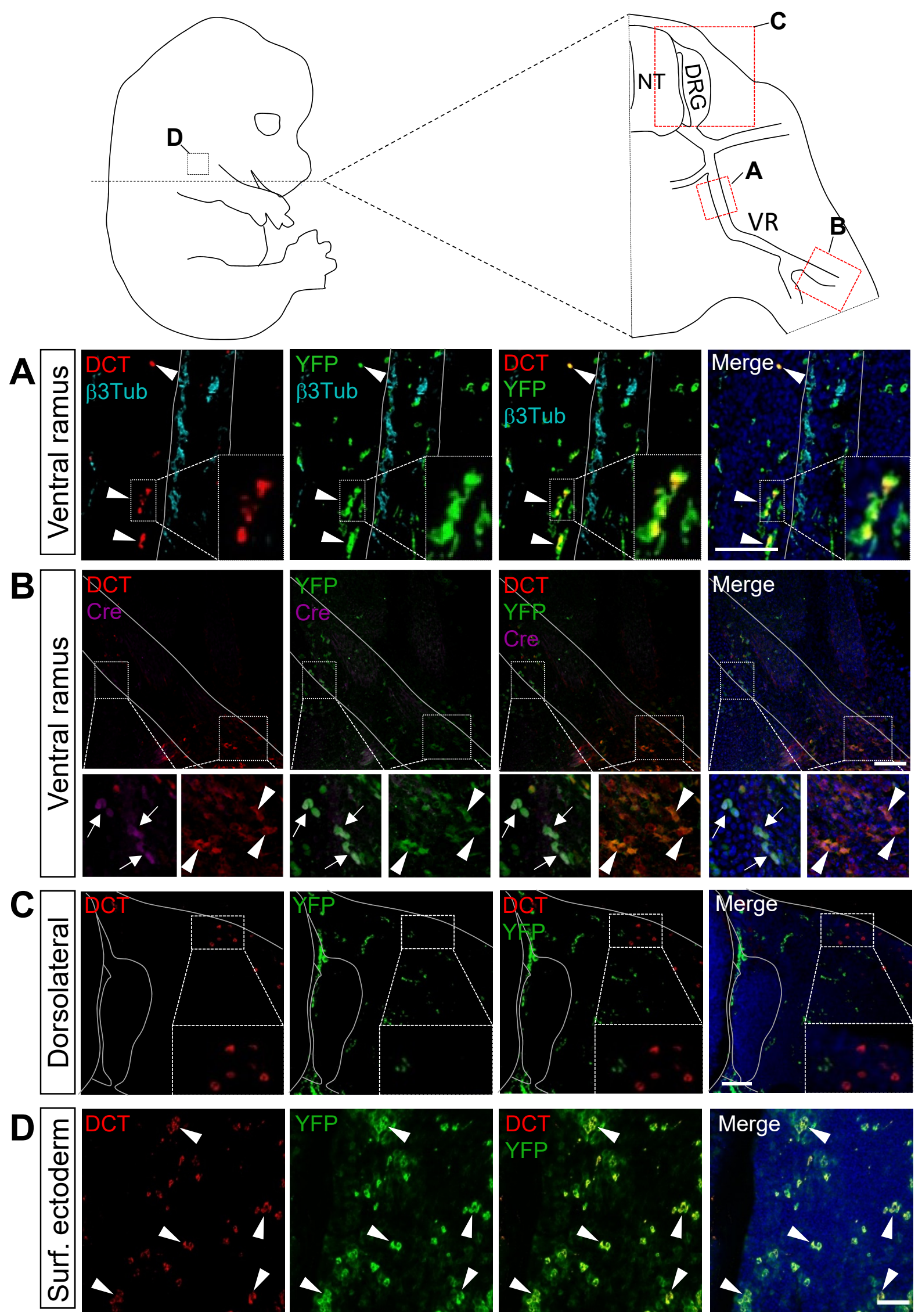


Figure 3
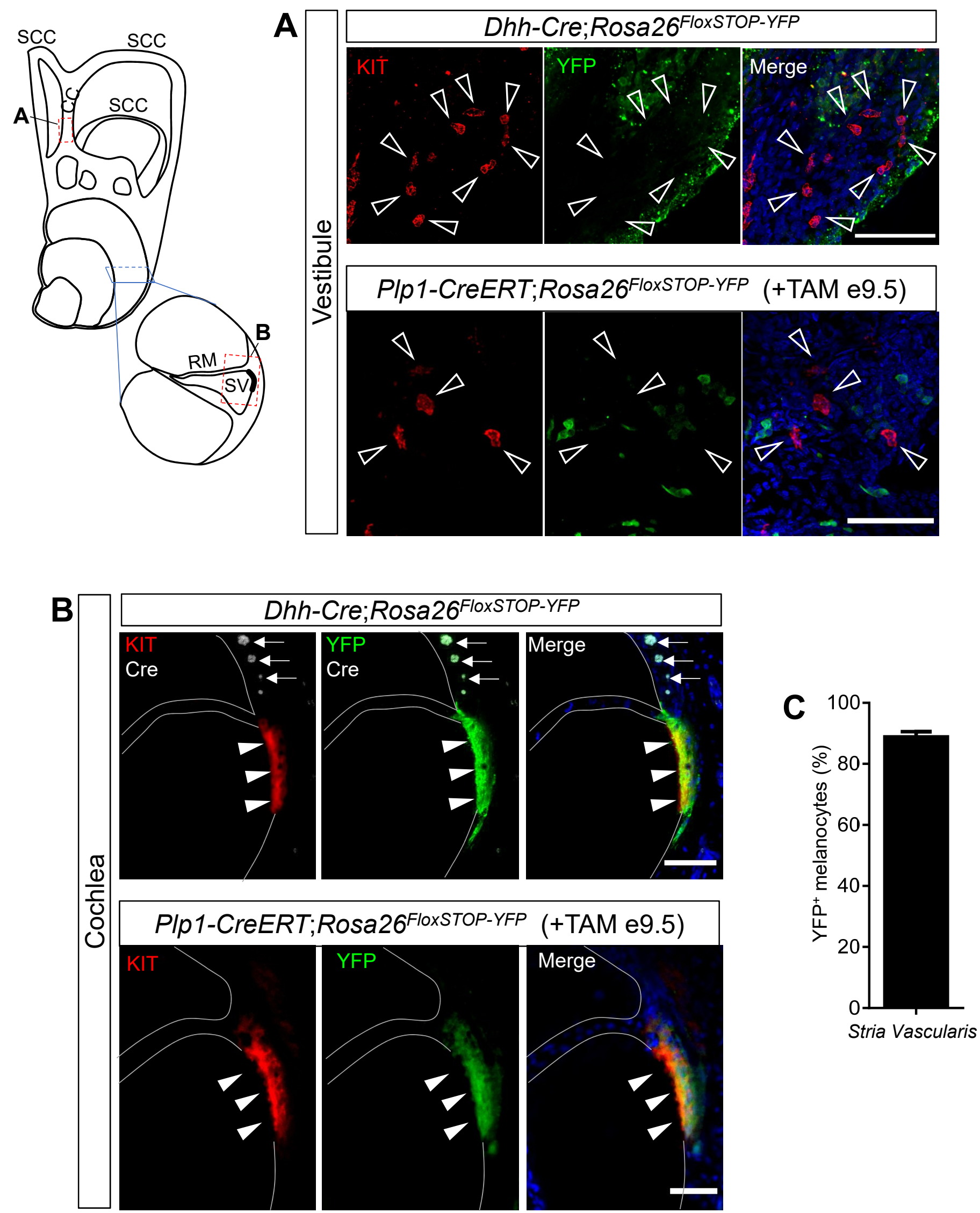